\title{
Evaluation of a Plasma Insulin Model for Glycaemic Control in Intensive Care
}

\author{
Jennifer Dickson, Felicity Thomas, Chris Pretty, Kent Stewart, Geoffrey Shaw, and J. Geoffrey Chase \\ Member, IEEE,
}

\begin{abstract}
Hyperglycaemia is a common complication in the intensive care unit (ICU), and is associated with worsened outcomes. Model-based insulin therapy protocols have been shown to be safe and effective in intensive care. Such protocols rely on correct modeling of glucose-insulin dynamics. In particular, model-based control typically relies on insulin sensitivity (SI) metrics, which are heavily influenced by plasma insulin kinetics. Plasma insulin samples were taken as part of a sepsis study and compared to modeled plasma insulin. Samples were taken in septic patients at the onset of glycaemic control, and once the patient consistently met less than two of the SIRs criteria that help define sepsis. It was found that inter-patient insulin dynamics were more variable at the onset of insulin therapy, than in the later samples after sepsis abated. Overall, the model adequately captured crucial steady state dynamics. Transient dynamics in plasma insulin following a bolus were faster than modeled, indicating greater clearance of insulin than currently modeled.
\end{abstract}

\section{INTRODUCTION}

Stress-induced hyperglycaemia is a common complication in the intensive care unit (ICU), even in patients with no history of diabetes [1-3], and is associated with increased mortality and morbidity [1, 4-9]. Insulin therapy can be used to treat hyperglycaemia, but can result in hypoglycaemia, which is associated with increased mortality [10], and results from excessive intra- and inter- patient variability. STAR is a physiological model-based insulin therapy protocol that has proven safe and effective in intensive care [11,12].

Model-based protocols rely on the inherent accuracy and usability of their models [13]. Key aspects of a glucoseinsulin system compartment model, when used in control, are the plasma and peripheral insulin concentrations. Insulin mediates glucose uptake into body cells, where it is stored or used. Insulin is cleared via liver and kidney clearance, as well as diffusion to interstitial fluid and cellular degradation [14]. Within the STAR model-based framework, an insulin sensitivity parameter is used to describe the time varying and

*Research supported by HRC, Christchurch, New Zealand.

Corresponding author: J.L Dickson is with the Department of Mechanical Engineering, University of Canterbury, Christchurch, New Zealand (Private Bag 4800, Christchurch 8140, NZ; email: jennifer.dickson@canterbury.ac.nz).

F.L Thomas, K.W Stewart, C.G Pretty, and J.G Chase are with the Department of Mechanical Engineering, University of Canterbury, Christchurch, New Zealand (e-mail: felicity.thomas@pg.canterbury.ac.nz, kent.stewart@pg.canterbury.ac.nz, chris.pretty@canterbury.ac.nz, geoff.chase@canterbury.ac.nz).

G.M Shaw is with the Department of Intensive Care, Christchurch Hospital, Christchurch, New Zealand, and also with the University of Otago, Dunedin, New Zealand (email: Geoff.Shaw@cdhb.health.nz). patient-specific metabolic effect of insulin on insulinmediated glucose uptake [15]. Thus, accuracy of plasma insulin dynamics has a significant impact of identified parameters such as insulin sensitivity (SI), and, in turn, on the recommended dosing.

In particular, insulin sensitivity is used alongside stochastic modelling methods to predict future changes in blood glucose for a given dose $[11,16]$. Thus, reduction of error in modelled plasma insulin can reduce SI variability and further increase the accuracy and utility of the parameter for glycaemic control. This paper evaluates the accuracy of insulin system models for intravenous (IV) insulin administration. Plasma insulin blood samples from a study of sepsis were used to evaluate these models.

\section{PATIENTS AND METHODS}

\section{A. Sepsis Study Patients}

19 patients enrolled in a prospective clinical trial studying sepsis at the Christchurch Hospital Intensive Care Unit (ICU) each had an additional two sets of blood samples assayed for insulin and C-peptide. Patients received insulin therapy (Actrapid, Actrapid, Novo Nordisk, Denmark) under the SPRINT protocol [17], a precursor to the STAR protocol. Patients were included in the study if they met all of the following criteria:

- $\quad$ Age $\geq 16$ years

- $\quad$ Expected survival $\geq 72 \mathrm{hrs}$

- $\quad$ Expected ICU length of stay $\geq 48 \mathrm{hrs}$

- Entry to the SPRINT glycaemic control protocol (2 sequential BG measurements $\geq 8 \mathrm{mmol} / \mathrm{L}$ )

- $\quad$ Suspected sepsis or SIRS score $\geq 3$

Patient characteristics are in Table I.

TABLE I. SUMMARY OF SEPSIS STUDY PATIENT CHARACTERISTICS. DATA ARE SHOW AS MEDIAN [IQR] WHERE APPROPRIATE.

\begin{tabular}{ll}
\hline \hline $\mathrm{N}$ & 19 \\
Age (years) & $68[57-75]$ \\
Gender (M/F) & $10 / 9$ \\
APACHE II score & $22.0[18.3-26.8]$ \\
Confirmed Sepsis & $79 \%$ \\
Hospital mortality (L/D) & $(13 / 6)$ \\
Diagnosed T2DM & 3 \\
\hline \hline
\end{tabular}


Patients received treatment for suspected sepsis with antibiotics. No type 1 diabetic patients were included. This study was approved by the Upper South Regional Ethics Committee, New Zealand.

One additional sepsis patient admitted to the ICU after pancreatoduodenectomy (Whipple procedure) was excluded from this analysis as this procedure involved removing a section of the pancreas and may thus have affected insulin secretion beyond model assumptions. Two other patients each only had one set of blood samples assayed as one was discharged from the ICU within 48 hours and the other did not meet the criteria for the second set to be taken.

Each patient had two sets of blood samples taken, where each set consisted of 4 separate samples. The first set of samples (Sample Set 1) was taken at the commencement of the SPRINT protocol [17]. The second set (Sample Set 2) was taken when the patient consistently met less than 2 of the SIRS criteria (Systemic Inflammatory Response Syndrome) [18].

The first sample of each set was taken immediately prior to bolus delivery of insulin as required by SPRINT $(\mathrm{t}$ $=-1 \mathrm{~min}$ ). The remaining three samples were taken at $\mathrm{t}=10$, 40, and 60 minutes. Plasma was separated from the blood samples and frozen for subsequent analysis.

Insulin concentrations were determined using immunometric assays (Elecsys 2010, Roche Diagnostics, Germany). The reported coefficients of variation $\left(\mathrm{CV}_{\mathrm{A}}\right)$ for the insulin assays were $3.8 \%[19,20]$.

\section{B. ICING Model}

For this study, the clinically validated Intensive Control Insulin-Nutrition-Glucose (ICING) model of the glucoseinsulin system was used $[11,15]$ to describe blood glucose, $G$, plasma insulin, $I$, and peripheral insulin, $Q$, concentrations:

$$
\begin{aligned}
& \dot{G}=-p_{G} G(t)-S_{I} G(t) \frac{Q(t)}{1+\alpha_{G} Q(t)}+\frac{P(t)+P N(t)}{V_{g}} \\
& +\frac{E G P_{b}-C N S}{V_{g}} \\
& \dot{I}=-\frac{n_{L} I(t)}{1+\alpha_{I} I(t)}-n_{K} I(t)-n_{I}(I(t)-Q(t))+\frac{u_{e x}(t)}{V_{I}} \\
& \dot{Q}=n_{I}(I(t)-Q(t))-n_{C} \frac{Q(t)}{1+\alpha_{G} Q(t)} \\
& u_{\text {en }}=\max \left(16.67, \frac{14 * G}{1+0.0147 * G}-41\right)
\end{aligned}
$$

Where $P$ and PN are glucose appearance from enteral and parenteral routes respectively. $u_{e x}$ is insulin introduced via IV bolus or infusion, and $u_{e n}$ is pancreatic insulin secretion. Further parameter descriptions and values can be found in Table 2.
TABLE II. ICING MODEL PARAMETER DESCRIPTION AND VALUES.

\begin{tabular}{ll} 
Variable Description & $\begin{array}{l}\text { Value and/or } \\
\text { units }\end{array}$ \\
\hline Endogenous glucose clearance & $0.006 \mathrm{~min}^{-1}$ \\
Insulin sensitivity* & $\mathrm{L} / \mathrm{mU} / \mathrm{min}$ \\
Insulin-mediated glucose uptake saturation & $1 / 65 \mathrm{~L} / \mathrm{mU}$ \\
Basal endogenous glucose production & $1.16 \mathrm{mmol} / \mathrm{min}$ \\
Central nervous system glucose uptake & $0.3 \mathrm{mmol} / \mathrm{min}$ \\
Distribution volume for glucose & $13.3 \mathrm{~L}$ \\
Liver clearance of insulin & $0.158 \mathrm{~min}^{-1}$ \\
Kidney clearance of insulin & $0.054 \mathrm{~min}^{-1}$ \\
Plasma $\leftrightarrow$ interstitial insulin diffusion & $0.0075 \mathrm{~min}^{-1}$ \\
Peripheral degradation of insulin & $0.0075 \mathrm{~min}^{-1}$ \\
First pass hepatic clearance of insulin & 0.67 \\
Saturation on liver clearance of insulin & $1.7 \times 10^{-3} \mathrm{~L} / \mathrm{mU}$ \\
Distribution volume for insulin & $4.0 \mathrm{~L}$ \\
\hline
\end{tabular}

*Insulin sensitivity is fit on a time varying, per-patient basis from measured BG data.

\section{Analysis of model accuracy}

Model error was analyzed in terms of the difference between measured and modeled insulin (vertical error) and perpendicular error. Vertical error is defined:

$E_{\text {vert }}=I_{\text {assay }}-I_{\text {modelled }}$

Perpendicular error is the smallest distance between a data point and the model, and better takes into account timing (horizontal) error in cases where the model gradient is very high. Perpendicular error is minimized a total least squares approach [21]. Perpendicular error is defined:

$E_{\text {perp }}=\min \left(\sqrt{\left(I_{\text {assay }}-I_{\text {modelled }, t n+i}\right)^{2}+\left(t_{\text {assay }}-t_{n+i}\right)^{2}}\right.$

Where $t_{n}$ is the assay time, and $t_{n+i}$ is the time corresponding to some nearby model solution. For each set of 4 samples the RMS vertical and perpendicular error was calculated.

To test sensitivity of insulin dynamics to clearance parameter values $n_{L}, n_{K}, n_{I}$ and $n_{C}$ were multiplied by a constant, $\xi$, which was allowed to range between 0.1 and 3.0. The intention was to find the $\xi$ which resulted in the best model fit with minimized perpendicular and vertical error.

\section{RESULTS AND DISCUSSION}

Measured sample results are shown in Table III. While plasma insulin was not significantly different $(p=0.11)$, plasma C-peptide concentration was much higher across Sample set $1(\mathrm{p}<<0.001$, Table III), indicating that insulin secretion was much higher in these samples, or clearance of C-peptide was lower but insulin clearance was not. This was also true when comparing C-peptide concentration across the first sample of each sample set (2225 [980-2735] vs. 799 [478 - 1000] $\mathrm{pmol} / \mathrm{L}, \mathrm{p}=0.002$ ), indicating that steady-state pre-insulin-bolus insulin secretion is higher when patients are septic. 
TABLE III: RAW RESULTS FROM EACH SAMPLE SET

\begin{tabular}{lccc}
\hline \hline & $\begin{array}{c}\text { Time since } \\
\text { TGC onset } \\
{[\text { hrs] }}\end{array}$ & $\begin{array}{c}\text { Insulin } \\
{[\mathbf{m U} / \mathbf{L}]}\end{array}$ & $\begin{array}{c}\text { C-peptide } \\
\text { concentration } \\
\text { [pmol/L] }\end{array}$ \\
\hline \hline Sample set 1 & - & $24.0[10.4-52.7]$ & $2050[993-2770]$ \\
Sample set 2 & $84[77-142]$ & $20.9[7.9-42.9]$ & $758[487-1052]$ \\
All & - & $20.9[8.6-51.4]$ & $1270[558-2345]$ \\
\hline \hline
\end{tabular}

Plasma insulin concentrations and model solution are shown in Figure I. It can be seen that in most cases the initial insulin clearance is faster than currently modeled. This result is also seen in Table II, where $\xi>1$ resulted in improved model fit across most of the samples. Across both initial and follow up sampling groups, the median [IQR] value of $\xi$ that was required to optimize model fit was $2.1[1.3-2.7]$, suggesting that generalized insulin clearance is twice as fast as the value originally modeled, and thus that one or more of the clearance dynamics is significantly faster than currently modeled.

In the original formulation of ICING model parameters, Lin et al used a combination of known C-peptide dynamics, and grid search over a likely physiological parameter range [15]. The grid search selected values such that the difference between modeled and measured $\mathrm{BG}$ was minimized. It is thus likely that, while these insulin clearance parameters are within a physiologically likely range, insulin dynamics may in reality be faster.

Model error was higher $(\mathrm{p} \leq 0.06)$ in the first set of blood samples, corresponding to the onset of tight glycaemic control in a septic patient, reflecting the higher inter-patient variability seen in plasma insulin clearance at this stage in a patient's infection state. Table III indicates that insulin was cleared faster at the time the first set of samples was taken (higher $\xi$ ). C-peptide results also suggest higher insulin secretion in the septic sample cohort, reflecting relative insulin resistance. This result seems to indicate that insulin dynamics are disease state dependant in their value.

The second set of samples were taken when a patient consistently met less than two of the SIRS criteria, reflecting improved patient condition. Lower model error in this set of samples indicates that in less ill patients insulin dynamics are more consistent between patients, and are thus more easily modeled. $\xi$ was also lower, indicating that insulin was cleared more slowly than in the first sample sets.
Model error in general was higher in the first half hour following the insulin bolus, where plasma insulin was more dynamic. The vertical error is greater than the $3.8 \%$ error associated with the immunoassay procedure. However, the difference between vertical and perpendicular error is high, indicating that slight offsets in time result in significant differences in insulin concentration due to large gradients in the modeled trajectory around a bolus. In this situation, perpendicular error gives a more informative reference for model error, as timing offsets between model and samples in samples are clinical reality due to a number of factors, such as timing differences in sampling and therapy.

In the case of the STAR protocol, the ability of the model to capture the steady state dynamics is more important than the ability to capture the first transient insulin peak following an IV insulin bolus. This priority occurs because, while transient plasma insulin contributes to initial BG drop, final $\mathrm{BG}$ at the end of an hour is more dependent on the steady state plasma insulin concentration. This final $\mathrm{BG}$ value is what drives the control protocol, and measured response based on infrequent $\mathrm{BG}$ measurements is more important than the transient trajectory in between those measurements.

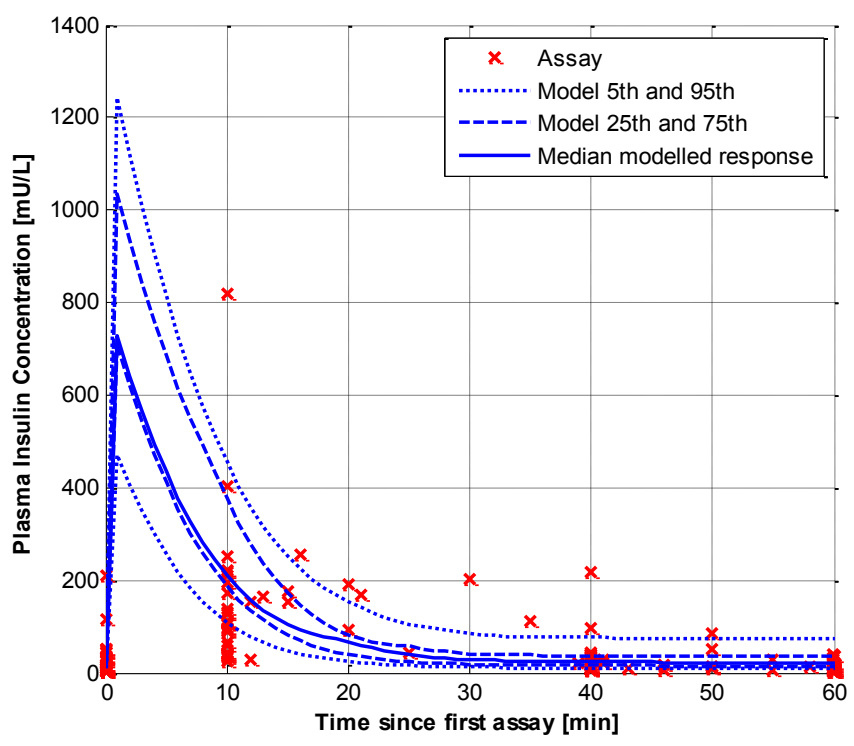

FIGURE I. ASSAY VALUES AND MODELED RESPONSE ACROSS ALL PATIENTS AND SAMPLING GROUPS.

TABLE IV: MODEL FIT TO INSULIN ASSAY DATA FOR DIFFERENT INSULIN CLEARANCE PRAMTERS

\begin{tabular}{|c|c|c|c|c|c|}
\hline & \multicolumn{2}{|c|}{$\xi=1.0$} & \multicolumn{3}{|c|}{ Minimum error } \\
\hline & $\begin{array}{c}R M S \\
\text { Vertical error } \\
{[\mathrm{mU} / L]}\end{array}$ & $\begin{array}{c}\text { RMS } \\
\begin{array}{c}\text { Perpendicular } \\
\text { error }\end{array} \\
\sqrt{[m U]^{2}+[\min ]^{2}}\end{array}$ & $\xi$ & $\begin{array}{c}\text { RMS } \\
\text { Vertical error }\end{array}$ & $\begin{array}{c}\text { RMS } \\
\text { Perpendicular error }\end{array}$ \\
\hline Sample set 1 & $202[116.2-454.3]$ & $24.8[18.9-71.7]$ & $2.4[1.1-2.7]$ & $158.6[64.6-318.9]$ & $16.0[11.7-31.2]$ \\
\hline Sample set 2 & 87.4 [101.1 -128.3] & $18.6[13.7-33.2]$ & $1.8[1.3-2.3]$ & $62.1[29.6-128.6]$ & $11.3[4.7-18.0]$ \\
\hline All & $123.7[89.2-234.7]$ & $22.7[15.4-37.9]$ & $2.1[1.3-2.7]$ & $97.1[43.5-192.5]$ & $13.3[9.3-19.8]$ \\
\hline
\end{tabular}


Blood samples were to be taken at $0,10,40$, and 60 minutes after an IV insulin bolus, but in some cases clinical workload and/or repeated sampling requirements resulted in a delay. Figure I shows that this sampling regime is insufficient to capture the initial data peak. A sampling regime of $0,5,10$, 20 and 60 minutes would better capture initial insulin dynamics.

In general, Figure I shows that the insulin kinetics of the ICING model fall within what might be clinically observed. Across most samples, insulin clearance was higher than currently modeled, and insulin clearance was highest at the onset of glycaemic control when the patients were most unwell. Inter- and intra- patient variability in the rate of insulin clearance (Table IV) reflects previously observed variability in human glucose-insulin physiology [22], and the need for adaptive control methods [23].

These results suggest that the insulin clearances within the model should be made faster. However, the ICING model must be generalisable to all patient cohorts across the ICU, unless clear condition or patient specific differences can be consistently noted at the bedside. Further work over other ICU patient cohorts and underlying disease conditions is required to develop more condition and time dependant modeling of clearance parameters. Overall, crucial steady state plasma insulin levels following an IV insulin bolus are captured.

\section{CONCLUSION}

Plasma insulin samples were taken in sepsis patients at glycaemic control onset, and once the patient met less than 2 of the SIRs criteria, a median of 84 hours later. The ICING model's insulin kinetic models were evaluated against these samples, and it was found that in general plasma insulin clearances were faster than currently modeled. Inter-patient variability was higher at the onset of glycaemic control. Model fitting error and insulin clearance was lower in the second set of samples. C-peptide concentration was higher in the first set of samples, with similar plasma insulin concentrations, suggesting that insulin secretion was higher when the patient was more ill, and relative insulin sensitivity was lower. These overall results suggest that insulin kinetics are condition dependant. Thus, it is critical to develop greater data sets and delineate the variation across common or particularly critical, such as sepsis, patient conditions. Capturing this variability in the insulin dynamics modeling will ensure that all other, already well-proven models remain fully generalisable.

\section{ACKNOWLEDGMENT}

Our thanks to the Christchurch Hospital Intensive Care Unit team for their help in collecting data.

\section{REFERENCES}

1. Capes, S.E., et al., Stress hyperglycaemia and increased risk of death after myocardial infarction in patients with and without diabetes: a systematic overview. Lancet, 2000. 355(9206): p. 773-778.
2. van den Berghe, G., et al., Intensive insulin therapy in critically ill patients. N Engl J Med, 2001. 345(19): p. 1359-67.DOI: 10.1056/NEJMoa011300.

3. McCowen, K.C., A. Malhotra, and B.R. Bistrian, Stress-induced hyperglycemia. Crit Care Clinics, 2001. 17(1): p. 107-124.

4. Holm, C., et al., Acute hyperglycaemia following thermal injury: friend or foe? Resuscitation, 2004. 60(1): p. 71-7.

5. Krinsley, J.S., Association between hyperglycemia and increased hospital mortality in a heterogeneous population of critically ill patients. Mayo Clin Proc, 2003. 78(12): p. 14711478.

6. $\quad$ Sperry, J.L., et al., Early hyperglycemia predicts multiple organ failure and mortality but not infection. J Trauma, 2007. 63(3): p. 487-93; discussion 493-4.DOI: 10.1097/TA.0b013e31812e51fc.

7. Hey, E., Hyperglycaemia and the very preterm baby. Semin Fetal Neonatal Med, 2005. 10(4): p. 377-87.

8. Heimann, K., et al., Are recurrent hyperglycemic episodes and median blood glucose level a prognostic factor for increased morbidity and mortality in premature infants $</=1500 \mathrm{~g}$ ? Journal of perinatal medicine, 2007. 35(3): p. 245-8.

9. Hall, N.J., et al., Hyperglycemia is associated with increased morbidity and mortality rates in neonates with necrotizing enterocolitis. Journal of pediatric surgery, 2004. 39(6): p. 898901; discussion 898-901.

10. Bagshaw, S., et al., The impact of early hypoglycemia and blood glucose variability on outcome in critical illness. Critical care, 2009. 13(3): p. R91.

11. Fisk, L., et al., STAR Development and Protocol Comparison. IEEE Trans Biomed Eng, 2012. 59(12): p. 3357-3364.DOI: 10.1109/TBME.2012.2214384.

12. Evans, A., et al., Pilot proof of concept clinical trials of Stochastic Targeted (STAR) glycemic control. Ann Intensive Care, 2011. 1(1): p. 38.DOI: 2110-5820-1-38 [pii]

10.1186/2110-5820-1-38.

13. Chase, J.G., et al., Physiological modeling, tight glycemic control, and the ICU clinician: what are models and how can they affect practice? Annals of Intensive Care, 2011. 1(11).DOI: doi:10.1186/2110-5820-1-11.

14. Duckworth, W.C. and A.E. Kitabchi, Insulin metabolism and degradation. Endocr Rev, 1981. 2(2): p. 210-33.

15. Lin, J., et al., A physiological Intensive Control InsulinNutrition-Glucose (ICING) model validated in critically ill patients. Computer methods and programs in biomedicine, 2011. 102(2): p. 192-205.DOI: 10.1016/j.cmpb.2010.12.008.

16. Lin, J., et al., Stochastic Modelling of Insulin Sensitivity Variability in Critical Care. Biomed Signal Process Control, 2006. 1: p. 229-242.

17. Lonergan, T., et al., A pilot study of the SPRINT protocol for tight glycemic control in critically Ill patients. Diabetes technology \& therapeutics, 2006. 8(4): p. 449-62.

18. Bone, R.C., et al., American-College of Chest Physicians Society of Critical Care Medicine Consensus Conference - Definitions for Sepsis and Organ Failure and Guidelines for the Use of Innovative Therapies in Sepsis. Crit Care Med, 1992. 20(6): $\mathrm{p}$. 864-874.

19. Roche, Data Sheet - C-Peptide Immunoassay, Elecsys 1010/2010/Modular Analytics E170, 2005, Roche Diagnostics, Mannheim, Germany.

20. Roche, Data Sheet - Insulin Immunoassay, Elecsys 1010/2010/Modular Analytics E170, 2004, Roche Diagnostics, Mannheim, Germany.

21. Tofallis, C., Model fitting for multiple variables by minimising the geometric mean deviation. Total Least Squares and Errorsin-Variables Modeling, 2002: p. 261-267.

22. Dickson, J.L., C.A. Gunn, and J.G. Chase, Humans are horribly variable. International Journal of Clinical and Medical Imaging, 2014. 1(2).DOI: 10.4172/ijcmi.1000142.

23. Le Compte, A.J., et al., Impact of variation in patient response on model-based control of glycaemia in critically ill patients. Computer methods and programs in biomedicine, 2013. 109(2): p. 211-9.DOI: 10.1016/j.cmpb.2011.08.007. 